\title{
An ethical reflection on doctor-patient relationship
}

Adhikari N

Intern, Kathmandu Medical College Teaching Hospital, Kathmandu, Nepal

B eing a doctor is a childhood dream of every student in Nepalese society where focus has always been to study hard and score more. Majority of us may have answered "to be a doctor" as our aim in life whenever someone asked us what we wanted to be in future. We have always imagined a doctor as a person wearing a white coat, donning a cheerful smile and caring personality.

Medicine indeed is a noble profession. It is serving mankind since the starting of human civilization and maintains its dignity for ages. Unlike other fields, medical profession is not just about dealing with the machine but an emotional and sensitive human being.

As Maimonides said, "The physician should not treat the disease but the patient who is suffering from it".

Nowadays many people are not happy with the care they are receiving in hospitals. My aunt once was complaining the way she was treated at one of the hospitals in Kathmandu. She had to wait for long hours without proper information on what she actually had to do and where she had to go for advice. The meeting was for few minutes when she finally got her turn. Coupled with hospital mismanagement, the doctor himself was very rude and was not answering her queries properly. A doctor is for patients and he is obliged to inform his patients. All she wanted was some more time and proper explanation with caring words. She advised me, "When you become a doctor, at least be kind to your patient".

Address for correspondence

Dr. Nimesh Adhikari

Intern

Kathmandu Medical College Teaching Hospital,

Sinamangal, Kathmandu, Nepal

E-mail: nimesh.adhikari@hotmail.com
We know the hectic schedule and busy life of doctors. It is not always possible to treat everybody in the same manner. They also have their arguments relating to the accusations they face every single day. But we have to remind ourselves of the Hippocratic Oath which every physician had taken swearing to practice medicine ethically and honestly. These basic moral principal today are not being followed properly and as a result ethical conflicts are rising. The best remedy to avoid disagreements might be through communication ${ }^{1}$.

I have not started practising as a doctor yet, but am interacting with patients during my clinical postings. This is the very place where we learn the technicalities and clinical skills. This is again the same place where we start to be responsible and sensible towards our patients. We can instantly notice a dramatic influence of our kind words on our patients. The gradual degradation of these sensitivities while we are busy accumulating the medical knowledge is creating a problem. We are so occupied in the process that we almost forget the humane treatment our patients deserve.

Our concern, care and few good words is a great support in their recovery. So we, as a medical student, should have a positive attitude right from the beginning. Apart from becoming a sound academic physician, try to be a nice DOCTOR, in a practical sense, like what we aspired to be in our childhood.

\section{REFERENCES}

1. Medical ethics [Internet].Importance of communication;Available from http://en.wikipedia. org/wiki/Medicalethics 